\title{
Investigación
}

\section{Prisión perpetua en Colombia: Funciones de la pena, política criminal y neuroderecho ${ }^{1}$}

\author{
Life imprisonment in Colombia: Functions \\ of punishment, criminal policy and neurolaw
}

Diego Alejandro Borbón Rodríguez*

Recepción: 28/07/2020 • Aprobación: 24/08/2020 • Publicación: 01/12/2020

Para citar este artículo

Borbón Rodríguez, D. A. (2020). Prisión perpetua en Colombia: Funcio-

nes de la pena, política criminal y neuroderecho. Dos mil tres mil, 22, e250.

https://doi.org/10.35707/dostresmil/22250

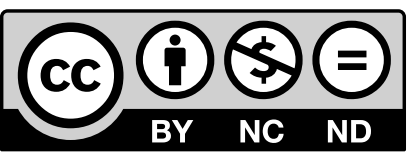

\footnotetext{
${ }^{1}$ El preprint de este artículo fue publicado en ResearchGate. Ver: Borbón Rodríguez, D. A. (2020, mayo).

* Universidad Externado de Colombia, Centro de Investigación en Política Criminal, Colombia. ORCiD: 0000-0002-2115-2105. Correo electrónico: diego.borbon01@est.uexternado.edu.co
} 
Resumen. El pasado 18 de junio de 2020, el Congreso de la República de Colombia aprobó en su octavo debate el Acto Legislativo 01 de 2020 que levanta la prohibición constitucional de la prisión perpetua. El presente artículo pretende abordar integralmente el estudio de la idoneidad de la prisión perpetua desde las funciones de la pena y el neuroderecho. Se encuentra dividido en dos secciones: la primera relaciona las funciones de prevención general, retribución justa y protección al condenado, la segunda sección contiene reflexiones sobre los mitos de la resocialización, su vínculo con la psicopatología y el neuroderecho. De esa manera, parecen existir las suficientes razones para afirmar que la prisión perpetua no satisface ninguna función de la pena.

Abstract. Last June 18, 2020, the Congress of the Republic of Colombia approved in its eighth debate the Legislative Act 01, 2020 that declares the constitutional ban of life imprisonment. This article aims to comprehensively address the study of the suitability of life imprisonment from the purposes of punishment and neuro-law. It is divided into two sections: the first one involves purposes of general prevention, fair compensation, and convicted protection; the second section contains reflections regarding myths of reincorporation, its link with psychopathology and neuro-law. In this matter, it seems to exist enough reasons to state that life imprisonment does not fulfill any purpose of the penalty.

Palabras claves

Colombia, prisión perpetua, neuroderecho, prevención, resocialización, retribución justa, protección al condenado.

Key words

Colombia, life imprisonment, neuro-law, prevention, reincorporation, fair compensation, convicted protection. 


\section{Introducción}

Para el sociólogo Nils Christie (1988), "el castigo, como lo impone el Código Penal, es la imposición consciente de dolor" (p. 20). No es complicado advertir, además, que el derecho penal es la herramienta jurídica más lesiva de derechos fundamentales con la que cuentan los Estados. La sanción suspende la libertad, pero también otra amplia lista de derechos, incluso, a tal extremo de acabar con la vida humana. Más aún, en las realidades de los sistemas carcelarios y penitenciarios, las condiciones de reclusión son degradantes e indignas. No en vano, en tres ocasiones la Corte Constitucional en Colombia ha declarado el estado de cosas inconstitucional, por la vulneración grave, sistemática, generalizada y masiva de derechos fundamentales de las personas privadas de su libertad ${ }^{2}$.

Si esto es así, si el derecho penal es la imposición intencional del dolor y dicha sanción lesiona y suspende un gran abanico de derechos fundamentales, vale preguntarse por los límites a ese dolor. Para Ferrajoli, aun cuando el derecho penal tiene grandes restricciones como las propias disposiciones, las garantías y derechos fundamentales, "conserva siempre una intrínseca brutalidad que hace problemática e incierta su legitimidad moral y política" (Ferrajoli, 1995, p. 21). En ese sentido, propone que la lógica de un modelo penal garantista equivalga a un sistema de minimización del poder y de maximización del saber judicial (Ferrajoli, 1995). En otras palabras, es necesario buscar un derecho penal mínimo, que sea utilizado como el último recurso, y que esté vinculado inescindiblemente a los principios de necesidad, proporcionalidad y razonabilidad ${ }^{3}$.

En Colombia, más allá de los límites de la Constitución Política como norma de suprema jerarquía, algunas de las llamadas "normas rectoras", en la parte general del Código Penal, se preguntan por la justificación de la sanción. En relación con esto, el artículo 4 del Código Penal colombiano (Ley 599 de 2000) establece las siguientes funciones de la pena: "La pena cumplirá las funciones de prevención general, retribución justa, prevención especial, reinserción social y protección al condenado. La prevención especial y la reinserción social operan en el momento de la ejecución de la pena de prisión".

Es particularmente interesante la forma en la que se redactó esta disposición, pues en nuestro concepto, no establece un fin de la pena como deber ser, sino que le otorga la función que cumplirá, es decir, el ser de la pena. De acuerdo con el Diccionario de la Lengua Española, fin significa "objeto o motivo con que se ejecuta algo" (RAE, s.f., s. p.); en otras palabras, lo que busca ser la pena, más no lo que es (Kelsen, 2019) ${ }^{4}$. En cambio, cuando el legislador consagra en el Código Penal colombiano que la pena cumplirá una función —cumplirá es la conjugación del futuro simple de la segunda persona singular de cumplir-, y función hace referencia a lo que en

\footnotetext{
${ }^{2}$ Véase las Sentencias T-153/1998, T-388/2013 y T-762/2015 de la H. Corte Constitucional de Colombia.

${ }^{3}$ El artículo 3 del Código Penal colombiano, Ley 599 del año 2000, establece que "La imposición de la pena o de la medida de seguridad responderá a los principios de necesidad, proporcionalidad y razonabilidad".

${ }^{4}$ Para Kelsen las normas prescriben un deber ser, precisamente porque pretenden influir en la conducta humana y cuando una norma expone que algo deba ser, no puede seguirse entonces que sea.
} 
concreto la pena es o será, no lo que persigue ser o representar ${ }^{5}$. Finalmente, de la redacción del artículo 4 se desprende que las funciones de prevención especial y reinserción social se cumplen al momento de ejecutar la pena, por exclusión; por lo tanto, las funciones de retribución justa y prevención general deberían cumplir su función al momento de la sentencia o incluso desde la formulación legislativa.

De esa forma, si la pena tiene una función que cumplirá, y no simplemente un objetivo abstracto, es relevante mencionar que el artículo 13 del Código Penal, dispone que las normas rectoras constituyen la esencia y orientación del sistema penal; prevalecen sobre las demás e informan su interpretación. En otras palabras, las funciones de la pena del artículo 4 no son simples enunciados sin fuerza normativa; son verdaderos mandatos para el sistema penal colombiano, para los operadores de justicia y para el legislador.

\section{Funciones de la pena y prisión perpetua}

El pasado 18 de julio de 2020, el Congreso de la República de Colombia aprobó, en su octavo y último debate, el Acto Legislativo que modifica el artículo 34 de la Constitución Política. Esto, con el fin de suprimir la prohibición que consagraba la Constitución para imponer la prisión perpetua. El nuevo texto constitucional será el siguiente:

[...] De manera excepcional cuando un niño, niña o adolescente sea víctima de las conductas de homicidio en modalidad dolosa, acceso carnal que implique violencia o sea puesto en incapacidad de resistir o sea incapaz de resistir, se podrá imponer como sanción hasta la pena de prisión perpetua.

Toda pena de prisión perpetua tendrá control automático ante el superior jerárquico. En todo caso la pena deberá ser revisada en un plazo no inferior a veinticinco (25) años, para evaluar la resocialización del condenado [...] (Congreso de la República, Acto Legislativo 01 de 2020).

El punto VI de la exposición de motivos del proyecto de Acto Legislativo consagra de manera expresa que "el aumento de la criminalidad hacia ciertos delitos y sujetos, hace necesario endurecer y mejorar los métodos para conseguir dicha finalidad" (Gaceta del Congreso del 26 de julio de 2019). Es por eso que una de las intenciones del legislador fue permitir la prisión perpetua para intentar prevenir la vulneración de los bienes jurídicos de los que son titulares los niños, niñas y adolescentes en Colombia a través de la amenaza de sanción severa.

En ese sentido, al analizar la hipótesis de prevención general negativa, según la cual elevar penas se traduce en poder disuasor dirigido a la sociedad o colectividad, se encuentran varias deficiencias. En el artículo de MacKenzie y Farrington (2015) analizaron ensayos controlados aleatorios, revisiones sistemáticas y metaanálisis en los últimos 10 años sobre la efectividad de las intervenciones correccionales, concluyen que la idea que sostiene que un período mayor de encarcelamiento disuadirá a las personas de futuras actividades delictivas, no parece estar respaldada por las investigaciones existentes. Incluso, por lo menos desde 1764, con la publicación

${ }^{5}$ En ese sentido, el artículo 61 de la Ley 599 de 2000 le otorga al juez la obligación de determinar el monto de la pena teniendo en cuenta, entre otras cosas, "[...] la necesidad de pena y la función que ella ha de cumplir en el caso concreto". 
de la obra Dei delitti e delle pene ${ }^{6}$, de Cesare Beccaria (2015), ya estaba claro que la severidad o gravedad de las penas no contiene o previene. Lo cierto es que la amenaza de sanción, por más grave que se ofrezca, no será idónea para evitar la desviación penal (Sánchez, 2010). El enfoque debería estar relacionado, en primer lugar, al estudio de las causas reales por las cuales se infringe la Ley penal, y en segundo lugar, para crear propuestas de política criminal que verdaderamente conjuren las razones estructurales de la desviación jurídico-penalmente relevante.

Usualmente se utiliza el concepto "populismo punitivo"7 para referirse a aquellas propuestas de mano dura contra el delito como, por ejemplo, elevar penas, crear nuevos tipos penales, reducir beneficios, limitar derechos y en general, cualquier otra propuesta para agravar las condiciones de reclusión (Romero y López, 2017). Si bien, formalmente, el derecho penal debe ser de ultima ratio, fragmentario y subsidiario (Gómez, 2016), infortunadamente, tal como lo afirma el Profesor Raúl Carnevali:

[...] ya no es posible siquiera hablar de ultima ratio sino que, derechamente, el Derecho penal es prima ratio, esto es, se emplea el recurso punitivo para prevenir ciertos comportamientos sin examinar siquiera cuan eficiente y necesario pueda ser este. Se afirma pues, que estamos insertos en un período de expansión del Derecho penal. (Carnevali, 2008, p. 31).

A manera de ejemplo, en Colombia, desde 1936 se ha aumentado por lo menos en un $168 \%$ el máximo de duración de la pena por el delito de homicidio simple, sin que por ello se pueda afirmar que se ha logrado reducir las cifras por ese delito (Gutiérrez, 2015). En específico, sobre reformas legislativas a aquellos delitos sexuales contra menores, desde el año 2000 al año 2008, la pena por el delito de acceso carnal abusivo con menor de catorce años aumentó un $200 \%$ en su mínimo y un $150 \%$ en su máximo punitivo; el delito de actos sexuales con menor de catorce años, aumentó en un $200 \%$ su mínimo y un $44 \%$ su máximo; y el delito de acceso carnal abusivo con incapaz de resistir recibió un aumento de $150 \%$ en sus dos extremos punitivos (Cita y González, 2017). A pesar de que se esperaría la disminución de las denuncias por delitos sexuales, desde el año 2009 al 2016, Pardo et al. (2019), reportaron que el histórico de exámenes de medicina legal por delitos sexuales, en lugar de reducirse, aumentaron. En fin, desde 2001, el Código Penal (Ley 599 de 2000) ha sido reformado en más de 50 ocasiones (Gutiérrez, 2017), se han creado más delitos, elevado penas, reducido beneficios; sin embargo, ninguna incidencia realmente cuantificable ha tenido estas modificaciones en la realidad. Incluso, cuando las estadísticas de la comisión de delitos bajan, no se puede afirmar que obedece a una relación de causalidad entre las medidas legislativas de mano dura y la reducción del crimen (Gutiérrez, 2015).

Si se pretende la prevención efectiva de los delitos contra niños, niñas y adolescentes, lo necesario para crear una política pública efectiva es valerse de los conocimientos que la criminología y la victimología nos aportan. De acuerdo con estadísticas del Instituto de Medici-

\footnotetext{
${ }^{6}$ De los delitos y las penas.

${ }^{7}$ Concepto incluso incorporado por la Corte Constitucional. Véase la sentencia T-762 de 2015: "A partir del diagnóstico presentado quedó claro que la política criminal colombiana se ha caracterizado por ser una respuesta al populismo punitivo".
} 
na Legal y Ciencias Forenses de Colombia, tan solo un 5,26 \% de los presuntos agresores por presuntos delitos sexuales son personas que la víctima no conocía. En cambio, el 47,8 \% de los presuntos agresores eran familiares, $23.06 \%$ conocidos, $8,51 \%$ amigos y 7,41 \% pareja o expareja. Por otra parte, de todos los exámenes médico-legales por presunto delito sexual, 87,45 $\%$ fueron practicados a menores de edad y de las 22309 valoraciones realizadas a mujeres por presunto delito sexual, 9545 entre los 10 y 17 años estaban embarazadas (Instituto Nacional de Medicina Legal y Ciencias Forenses, 2018).

La implementación de la cadena perpetua en nada contribuiría para solucionar las causas estructurales ni abordar integralmente la solución. En ese sentido, bien exponen las investigadoras del Centro de Investigación en Política Criminal, Pardo, Moncayo y Olarte:

La cadena perpetua no estaría enfocándose en el problema y estaría invisibilizando las causas y los factores de riesgo que requieren un abordaje integral, hacia la protección de las familias y la construcción de políticas preventivas orientadas al bienestar de toda la población, en especial los niños, las niñas, los adolescentes y las mujeres. (Pardo et al., 2019, p. 23).

La Defensoría del Pueblo de Colombia, en su informe "Violencias basadas en género y discriminación", analiza conjuntamente información sobre situaciones de violencia y acciones institucionales entre 2014-2018, en conjunto con organizaciones de la sociedad civil, entidades estatales y organismos internacionales. La Defensoría propone una serie de medidas integrales, tales como:

Deconstruir a través de la educación y la práctica institucional estereotipos machistas, prejuicios socioculturales, e imaginarios, que reproducen la violencia contra las mujeres y personas. Desarrollar políticas que respondan a las particularidades y necesidades de las mujeres en su diversidad: niñas, adolescentes, adultas mayores, campesinas, indígenas, afrocolombianas, Rom, lesbianas, bisexuales y trans. Trabajar en la construcción de nuevas masculinidades. Fortalecer los programas que inciden en la cultura colombiana. Generar mecanismos que permitan identificar la violencia psicológica, atendiendo y reportando de manera adecuada. Robustecer el presupuesto asignado a la erradicación de la violencia y discriminación basada en género. Facilitar la denuncia por hechos relacionados con violencia y discriminación basada en género. Investigar, procesar y sancionar, dentro de un plazo razonable a los responsables de actos de violencia basada en género. Generar alianzas con el sector privado con el fin de involucrar a las empresas en la prevención de la violencia basada en género. Hacer un llamado a los medios de comunicación para que reconozcan el impacto que tienen en la transformación de la sociedad y se involucren en campañas para erradicar la violencia y la discriminación basada en género. Dirigir esfuerzos del Estado para fortalecer la presencia institucional en las zonas rurales. Adelantar acciones para mitigar el riesgo de violencia sexual, trata de personas, amenazas y homicidios. (Defensoría del Pueblo, 2018, p. 33).

A manera de síntesis, si se busca prevenir efectivamente los delitos contra niños, niñas y adolescentes, es necesario abordar las causas estructurales desde la criminología y la victimología, para poner sobre la mesa verdaderas propuestas de política criminal integral. Los proyectos 
como la prisión perpetua, lo único que logran es enviar un mensaje simbólico de que algo se está haciendo frente a los fenómenos de opinión pública. Sin embargo, ningún efecto tendría en la práctica y en la protección de la infancia. En un mismo sentido, pone en peligro la naturaleza humanista de la Constitución Política que reconoce la dignidad humana y proscribe la posibilidad de aplicar la cadena perpetua.

Es más, una propuesta de esta naturaleza solo podría sacrificar, en nombre de la prevención general negativa, los principios del derecho penal moderno, como la necesidad, proporcionalidad y ultima ratio. La Corte Suprema de Justicia de Colombia, en proceso de junio de 2011 n. ${ }^{\circ} 31895$ con magistrado ponente José Leonidas Bustos, expresó su preocupación por las recurrentes propuestas políticas que en ese tiempo planteaban implementar medidas como la cadena perpetua o la pena de muerte:

No obsta para que la Corte deje de expresar su preocupación por el desmesurado incremento de penas para algunos delitos, a punto de escucharse algunas voces que, aun en contravía de los compromisos internacionalmente adquiridos por Colombia, propugnan por el establecimiento de la cadena perpetua o la pena de muerte por la realización de determinadas conductas, pero sin atender para nada principios inherentes al derecho penal, tales como los relacionados con la naturaleza e importancia de los bienes jurídicos que se pretende tutelar, o con la gradualidad, proporcionalidad, racionalidad, utilidad y efectividad de las consecuencias legales por su lesión o puesta en peligro, aspectos que, como es apenas obvio, deben ser objeto de consideración al momento de determinar la clase e intensidad de las penas correspondientes a la naturaleza y gravedad social y jurídica de los delitos cometidos, máxime si se concibe el ordenamiento como un sistema coherente de disposiciones y no como algo irracional derivado del capricho del órgano legisferante, del gobernante de turno o del aparato judicial. (Corte Suprema de Justicia, proceso n. ${ }^{\circ} 31895$ de 2011).

La llamada función de prevención general negativa hace referencia precisamente a que la pena busca cumplir una función intimidatoria. Pero, por otra parte, existe también la función positiva de la prevención general, principalmente a la vanguardia con la teoría del funcionalismo normativo de Jakobs. Así entonces, el derecho penal persigue el fin de comunicar a los ciudadanos que la norma está vigente; en otras palabras, cuando una persona quebranta la norma, pone en duda las expectativas normativas de los demás ciudadanos, ergo, el derecho penal debe sancionarlo para reivindicar la vigencia del mismo. Se podría entender, por lo tanto, como la negación contra-fáctica: el delincuente niega al derecho, el derecho, entonces, debe negarlo a él y así estabilizar y restaurar la vigencia de la norma (Montealegre y Perdomo, 2006).

La función de negación contra-fáctica y la restauración de la expectativa normativa, por más teóricamente provocador que suene, puede ponerse especialmente en duda en sociedades con altas tasas de impunidad. De acuerdo con cifras de la Fiscalía General de la Nación, la tasa de esclarecimiento promedio a nivel nacional a noviembre de 2019 era de 11,88 \%. Segregando los datos por seccionales funcionales de la Fiscalía, se tiene que la seccional Boyacá de la Fiscalía presentó para noviembre de 2019 una tasa de esclarecimiento del 7,12\%, mientras que 
la seccional con mayor tasa de esclarecimiento en el país apenas llega al 36,61 \%, siendo esta la seccional del Caquetá (Fiscalía General de la Nación, 2019).

En ese sentido, no existe estadísticamente la certeza de que efectivamente se sancionarán los delitos que oficialmente se denuncian ante la Fiscalía. Más aún, ¿ cómo será posible restaurar una expectativa normativa y reivindicar la vigencia de la norma, cuando la tasa de esclarecimiento de los delitos sexuales apenas alcanza el 11,88 \%? Si acaso es cierto que el sistema se basa en expectativas normativas, y que el papel de la imposición de la pena es comunicarles a los ciudadanos la vigencia de la norma (Montealegre y Perdomo, 2006); sin pena, sin certeza de sanción y con el quebrantamiento reiterado y permanente en el tiempo de las expectativas normativas, no existe una función de prevención especial positiva. La expectativa normativa está y seguirá quebrantada. La prisión perpetua, en ese sentido, no se enfoca en lograr efectiva justicia, sino que deja intacta la estructura investigativa y juzgadora fallida del Estado colombiano. Hasta tanto no se fortalezca el sistema judicial mediante una verdadera política pública integral, la función de prevención especial positiva no será más que letra muerta.

Una vez analizado de manera sucinta el no cumplimiento de la función preventivo general, se analizará la función de "retribución justa", no sin antes advertir que resulta ser especialmente una discusión filosófica del concepto de retribución o de justicia, más que una utilidad práctica. Incluso, las teorías retributivas, entendidas como teorías absolutas, no buscan una función utilitarista, como podría ser, prevenir delitos. Estas se fundamentan en valores absolutos como la justicia, o la imposición de una pena por el simple hecho de infringir la Ley penal.

La primera consideración que debe tomarse en cuenta al analizar una propuesta de esta naturaleza es la no negación de la dignidad humana. Sin importar si es revisable o no, consideramos que la prisión perpetua niega tajantemente la existencia de la dignidad humana al plantear al ser humano no como un fin en sí mismo, sino como un medio, por ende, como sujeto carente de valor. No puede ser, desde ningún punto de vista, constitucionalmente legítimo que una persona condenada pierda su valor y carácter de persona digna, sin distinción del tipo de comportamiento cometido o su gravedad. La Corte Constitucional de Colombia, en Sentencia T-208 de 2018, estableció claramente que:

Como consecuencia de su comportamiento antisocial anterior, en caso de haber sido condenados o por existir una conducta en investigación, tienen algunas de sus garantías suspendidas, como la libertad, otras limitadas, como la comunicación o la intimidad, pero también gozan del ejercicio de bienes fundamentales básicos en forma plena, como la vida, la salud, la integridad física y la dignidad humana. Se trata de contenidos superiores dotados de poder para demandar del Estado su efectiva protección sin restricción alguna. (Corte Constitucional, T-208 de 2018).

La teoría del llamado "retribucionismo penal" encuentra a Kant, al parecer, como un importante exponente, partiendo de las bases del contrato social. Así, cuando una persona delinque, está aceptando la pérdida de su libertad al negar el contrato social. Kant (1996), sostenía que la Ley penal es un imperativo categórico. Esto podría entenderse especialmente como 
principio de legalidad; esto es, el juez está obligado a aplicar la Ley penal. Así, se sostiene la importancia del llamado principio de igualdad, que, para Kant, podría acoplarse al talión: "Si deshonras, te deshonras a ti mismo; si le robas, te robas a ti mismo; si lo maltratas o lo matas, te maltratarás o te matarás a ti mismo" (Cordini, 2014, s. p.).

Resulta por lo más llamativo que el concepto de dignidad humana, adoptado por la Corte Constitucional en Colombia, proviene del mismo Kant. Este es, tratar al ser humano como un fin en sí mismo y no como un medio (Corte Constitucional, Sentencia C-143 de 2015). Precisamente por eso, para Kant, la pena se impone por el simple hecho de haberse infringido la Ley. No puede existir, para Kant, alguna finalidad de prevención para la sociedad, porque ello sería utilizar al ser humano como un medio para intimidar, no como un fin en sí mismo (Bacigalupo, 2010).

El derecho penal contemporáneo no puede ser entendido cercano al talión. El derecho punitivo es, incluso, un rechazo a la posibilidad del talión. Ferrajoli (1995) expone la llamada doble función preventiva del derecho penal; esto es, no solo procurar que los ciudadanos no delincan sino, además, contener las ansias del poder estatal y de venganza por propia mano. Por consiguiente, el derecho penal para Ferrajoli debe propender no solo por disuadir sino para la prevención de las penas arbitrarias o desproporcionadas:

La tutela del inocente y la minimización de la reacción al delito, sirve para distinguir el derecho penal de otros sistemas de control social - de tipo policial, disciplinario o incluso terrorista- que de un modo más expeditivo y probablemente más eficiente serían capaces de satisfacer el fin de la defensa social respecto al que el derecho penal, más que un medio, es por consiguiente un coste, o si se quiere un lujo propio de sociedades evolucionadas. (Ferrajoli, 1995, p. 334).

El derecho penal, en ese sentido, debe estar muy lejos de la venganza o del talión como su expresión de estricta proporcionalidad. Fundar un sistema penal en alguno de estos dos conceptos, es construir el aparato punitivo del Estado bajo instintos primitivos. E incluso, está claro que la prisión perpetua no representa la imposición estrictamente idéntica al mal causado, por lo que no ha lugar al argumento que intenta asemejar este tipo de penas a la proporcionalidad -irracional- del talión.

Por otra parte, el concepto de justicia resulta especialmente interesante. Por justicia pueden entenderse diversas cosas. Michael Sandel, de la Universidad de Harvard, escribe en su libro Justicia. ¿Hacemos lo que debemos?, que por lo menos existen tres grandes categorías del concepto de justicia: bienestar, libertad y virtud (Sandel, 2011). Nuestra opinión es que, más allá de los subjetivos conceptos de justicia, puede ser que el hacer justicia o, la decisión justa, se desprende de la aplicación de las normas al tomarse una decisión judicial; en ese sentido, independientemente del quantum de la pena, se hace justicia. Siguiendo a Ross:

Las palabras 'justa' e 'injusta' (o 'correcta' e 'incorrecta') tienen sentido cuando se aplican para caracterizar la decisión hecha por un juez, o por cualquier otra persona que debe aplicar un conjunto 
determinado de reglas. Decir que la decisión es justa significa que ha sido hecha de una manera regular, esto es, de conformidad con la regla o sistema de reglas vigentes. (Ross, 1994, p. 267).

Por otra parte, formalmente y en la praxis, es relevante mencionar que en Colombia existen ya penas de prisión de por vida. El artículo 60 del Código Penal establece que: "En ningún caso, en los eventos de concurso, la pena privativa de la libertad podrá exceder de sesenta (60) años". Si la expectativa de vida promedio para ambos sexos en Colombia es de alrededor de 76,15 años (Ministerio de Salud de Colombia, 2019), y la edad de imputabilidad en Colombia se ubica en los 14 años, ya existe en Colombia una prisión perpetua no reconocida legalmente. Además, es necesario tener en cuenta que será a partir de los 18 años que la persona será juzgada con el procedimiento ordinario, en el que le podría ser aplicable la pena máxima en caso de concurso de delitos. Bajo esa lógica, si lo que se busca es relacionar la reclusión hasta el fin de la vida, con la justicia y la retribución, ya existe una disposición normativa que logra esto.

Lo anterior es, sin lugar a duda, un grave error del legislador, pues a pesar de que formalmente no la consagra como prisión perpetua, una pena que puede llegar a ser de 60 años es en la práctica una pena perpetua. Aún más, la irracionalidad de la política penal formal llega al punto de que para ciertos delitos el quantum de la pena es mayor al límite legal de la parte general del Código Penal. Tómese tan solo el delito de lavado de activos agravado, cuya pena máxima puede ser de hasta 118,12 años (Cita y González, 2017).

Quizás el debate de la justicia nos debería conducir a reflexiones sobre las condiciones de reclusión en Colombia. Las sentencias T-153/1998, T-388/2013 y T-762/2015, declaran y reiteran la existencia de causas estructurales que derivan en la vulneración sistemática, masiva, grave y generalizada de todos los derechos fundamentales de las personas privadas de su libertad. Podría cuestionarse, por consiguiente, ¿es justicia la reclusión hasta la muerte bajo condiciones indignas y degradantes? Más aún cuando en Colombia oficialmente existen establecimientos de reclusión donde el privado de su libertad es sometido a condiciones de hacinamiento superiores al 300 \% (INPEC, 2020). Incluso, con la Sentencia T-151 de 2016, la Corte Constitucional corroboró que en las cárceles y penitenciarías de Colombia se vulnera el derecho a no ser sujeto de tortura, penas crueles, degradantes e inhumanas. En ese sentido, tanto el legislador como los operadores de la justicia penal deben tener claro que cualquier pena impuesta en nuestro país representa en sí misma una condena para que el sujeto sea sometido a tratos crueles e inhumanos, lo que a la luz de los planteamientos kantianos sería una negación directa del imperativo categórico de tratar al otro como un fin en sí mismo, así como una vulneración a la naturaleza humanista de la Constitución Política.

La Corte IDH, en el año 2017, actualizó el noveno cuadernillo de jurisprudencia sobre personas privadas de libertad. Recopila en más de 120 páginas varios títulos y resúmenes de sentencias judiciales en los que los Estados miembros son condenados como responsables de vulnerar la Convención Americana de Derechos Humanos y las normas internacionales de reclusión digna (Corte IDH, 2017). En varias de estas sentencias, por someter a las personas 
privadas de su libertad a condiciones indignas de reclusión por hacinamiento o pésima salubridad, se ha condenado a los Estados a sumas de más de 100000 dólares por cada persona afectada.

Las teorías absolutas de los fines de la pena señalan que la pena se agota en sí misma. Si la Ley penal es un imperativo categórico, y su aplicación equivale a la realización de la justicia, las leyes ya existentes constituirían justicia como materialización del principio de legalidad. En nuestra opinión, la cadena perpetua no brinda más justicia que las normas vigentes, ni podríamos decir que se configura como retribución más válida que la que teóricamente representan las penas actuales. Por otra parte, si se acepta la teoría pura de la retribución justa como función de la sanción, sería incompatible con plantear que se busca proteger y prevenir, bajo el entendido que no es posible buscar un fin utilitarista al imponer la pena. Nuevamente, el concepto de dignidad humana de Kant vuelve al escenario al plantear que el ser humano que es penado no lo puede ser para configurarse como instrumento para disuadir a la colectividad. Finalmente, está claro que las condiciones actuales de reclusión son abiertamente incompatibles con la dignidad humana y un gran abanico de derechos y garantías constitucionales, por esto tampoco es dable predicar un valor de justicia.

Lo anteriormente mencionado, sobre la función de retribución justa y, en especial, la mención sobre la injusticia que representa estar recluido en las condiciones degradantes e indignas del sistema carcelario y penitenciario, se vincula estrechamente al incumplimiento con la función de protección al condenado del artículo 4 del Código Penal. Las prisiones en Colombia representan los lugares menos idóneos materialmente existentes para privar de su libertad a una persona, mientras se pretende al mismo tiempo protegerla. Esto es aún más vigente bajo la coyuntura actual por la pandemia del coronavirus. Antes de la pandemia, eran 112 los establecimientos que contaban con un nivel de hacinamiento superior al $20 \%$, de estos, 86 con cifras mayores al $50 \%, 30$ presentaron niveles superiores al $100 \%, 7$ sobrepasan el $200 \%$ y 3 establecimientos de reclusión alcanzan estadísticas oficiales superiores al $300 \%$ de hacinamiento (INPEC, 2020). Lo anterior, incluso, fue objeto de manifestación por parte de la Comisión Interamericana de Derechos Humanos que mostró:

[...] su profunda preocupación por las alarmantes condiciones en las que se encuentra la población carcelaria en la región, que incluye precarias condiciones de salubridad e higiene y niveles de hacinamiento extremos, destacándose que en algunos países la tasa de ocupación es superior al $300 \%$. (CIDH, 2020, s. p.).

Por otra parte, la cidH destacó los preocupantes motines registrados en 13 cárceles de Colombia, en particular "los hechos ocurridos en la cárcel La Modelo de Bogotá donde fallecieron 23 personas y más de 80 resultaron heridas el 21 de marzo" (СIDH, 2020, s. p.). Así, no es complicado advertir que, en espacios de extremo e inaceptable hacinamiento, la sanción deja de ser una pena justa y protectora para representar nada más que una pena capital. Lo que las actuales 
condiciones de reclusión representan es, específicamente, la tortura, la degradación humana y la muerte.

Al 4 de noviembre de 2020, la cifra de contagios por covid-19 ascendía a 1532 personas privadas de su libertad positivos y 15247 PPL recuperados de la enfermedad (INPEC, 2020). Por otra parte, de los 132 establecimientos de reclusión, 43 presentaban casos activos (INPEC, 2020). A pesar de que la cifra de muertos por la enfermedad no está publicada en la página del Instituto Nacional Penitenciario y Carcelario, vía derecho de petición de información se pudo constatar que, al 9 de septiembre de 2020, por lo menos 77 personas privadas de su libertad habían perdido la vida (INPEC, Petición con radicado No. 2020ER0105109, 2020). Para el futuro, sobre el resultado de esta crisis humanitaria en las cárceles y penitenciarías, tal como lo auguraba la Corte Constitucional en 1998, "la reconstrucción de esta realidad dolorosa le corresponderá quizás a la literatura despiadada acusadora de las sociedades ante la historia" (Corte Constitucional, Sentencia T-153 de 1998).

\section{Función resocializadora de la pena, psicopatología y neuroderecho}

En la exposición de motivos del Acto Legislativo de prisión perpetua se hizo expreso, frente a la función resocializadora de la pena, que:

$\mathrm{Al}$ establecer que la medida tendrá que ser revisada en el término que señale el legislador, se está garantizando la función resocializadora de la pena, ya que esa revisión garantizará que en el caso de que se haya logrado la resocialización del individuo de manera cabal, el mismo pueda ingresar nuevamente a la sociedad rehabilitado plenamente. (Gaceta del Congreso del 26 de julio de 2019).

Usualmente se pretende justificar la prisión perpetua, al señalar que quienes cometen delitos sexuales graves padecen trastornos mentales y son incurables. Precisamente, durante las discusiones legislativas en el Congreso de la República, la mención de argumentos como estos fue recurrente ${ }^{8}$. Esta narrativa, muy común en los discursos políticos o informales, no podría estar más lejos de la realidad. Incluso, al seguir el MacArthur Violence Risk Assesment Study del año 1998, los actos violentos cometidos por personas que salen de hospitales psiquiátricos no son mayores a los que comete la comunidad en general (MacArthur, 1998). En general, es muchísimo más probable que las personas con trastornos mentales sean víctimas de violencia, y no sus perpetradores (Stuart, 2003). Por otra parte, a nivel global, hasta el $70 \%$ de las personas con trastornos mentales no reciben tratamiento; los estigmas y la discriminación hacia las personas que padecen enfermedades de salud mental contribuyen al aumento de esta brecha de atención psicológica y psiquiátrica (Henderson, Evans-Lacko y Thornicroft, G., 2013). Por

\footnotetext{
${ }^{8}$ Las discusiones pueden ser encontradas en los canales de YouTube oficiales del Senado y la Cámara de Representantes de Colombia. A manera de ejemplo, en séptimo debate, 9 de junio de 2020, el H.S Eduardo Enríquez menciona: "Estos delitos son cometidos por unos sociópatas, estas personas sin alma, deshumanizados", haciendo posterior alusión a la clasificación de delincuentes de Ferri, los criminales natos y la necesidad de retomar estos conceptos.
} 
otra parte, en Estados Unidos, menos del 40 \% de las personas con trastornos mentales severos recibieron tratamiento consistente. Estas personas reportaron en un $52 \%$ que no recibían tratamiento por barreras situacionales, $46 \%$ por barreras financieras y $45 \%$ por la falta de efectividad del tratamiento otorgado (Kessler et al., 2001). Es menester, por consiguiente, partir de la base de desestigmatizar y abandonar el lenguaje peyorativo que se emplea al hacer referencia a los padecimientos de salud mental. En segundo lugar, es imperativo mejorar la calidad y el acceso a los servicios públicos y privados de atención en salud mental.

Distintas investigaciones señalan resultados variables sobre el vínculo entre trastornos mentales y criminalidad sexual. Por una parte, al revisar la literatura disponible, se sugiere que los trastornos mentales graves solo tienen una pequeña incidencia en los delitos sexuales; en ese mismo sentido, el trastorno mental no se incluye entre los factores de riesgo para la comisión de violaciones sexuales según los estudios epidemiológicos, o el abuso sexual, en los estudios de revisión (Martins et al., 2013). Por otra parte, en una muestra significativa de personas de Dinamarca, se relacionó a los trastornos psicóticos comórbidos con los trastornos de la personalidad y los trastornos por uso de sustancias con un mayor riesgo de delincuencia sexual con y sin agresión física. En específico, los trastornos psicóticos junto con trastornos de personalidad o trastornos por uso de sustancias se asociaron con un riesgo 6 veces mayor de delincuencia sexual físicamente agresiva. Ahora bien, en un estudio con 134 delincuentes sexuales masculinos, las parafilias fueron el mayor diagnóstico: 76,9 \% eran pedófilos. Por otra parte, $29,1 \%$ de ellos presentan el trastorno de la personalidad antisocial, 20,1 \% trastorno de personalidad no especificado y 22,4 \% presentaron trastorno por abuso de alcohol (McLawsen et al., 2012).

La criminología como el estudio del porqué del delito, ha hecho especial énfasis al analizar la psicopatía o los trastornos de personalidad. Lo primero que resulta relevante es que el concepto de psicopatía es difuso y no existe unanimidad de su definición. Etimológicamente la palabra psicopatía está conformada por el griego phsyche (alma, actividad mental) y pathos (emoción, sentimiento, sufrimiento); es decir, patología de la mente, al fin y al cabo, nada específico. Robert Hare, reconocido psicólogo, popularizó el PCL, o Psychopathy Check List y su versión revisada PCL-R. Dicha herramienta psicométrica de diagnóstico constaba inicialmente de 22 puntos (luego 20, en la versión revisada), entre ellos: egocentrismo, mentira, manipulación, irresponsabilidad, impulsividad, falta de empatía, entre otros (Hare, 1991). La psicopatía como diagnóstico no existe en los manuales internacionales como el cIE (Clasificación Internacional de Enfermedades) o el DSM (Manual Diagnóstico y Estadístico de Enfermedades Mentales).

Resulta distinto el trastorno de la personalidad antisocial, reconocido como tal desde 1980 en el DSM-III. Actualmente permanece como diagnóstico en la quinta versión del DSM de la Asociación Americana de Psiquiatría. Robert Hare afirma que la mayoría de los psicópatas cumplen los criterios para el trastorno de la personalidad antisocial, pero la mayoría de las personas con dicho trastorno no son psicópatas (Hare, 1996). La Asociación Psiquiátrica Americana (APA), reseña que entre un $0,2 \%$ y un 3,3 \% de las personas en la población general podrían cumplir 
los criterios diagnósticos de este trastorno mental (APA, 2014). Sin embargo, la prevalencia de este tipo de trastornos en prisiones puede ser del $15 \%$ hasta el $47 \%$ (Pérez, 2015). Salta a primera vista la evidente relación estadística que parece tener este trastorno de personalidad y estar privado de la libertad en prisiones. De la misma manera, se relaciona este diagnóstico con la alta reincidencia después del cumplimiento de la pena, así como gran parte de la bibliografía resalta la dificultad que resulta tratar psicológicamente a personas con este diagnóstico (Pérez, 2015).

El trastorno de la personalidad antisocial está clasificado en el numeral 301.7 del DSM-V y establece los siguientes criterios diagnósticos:

A. Patrón dominante de inatención y vulneración de los derechos de los demás, que se produce desde los 15 años de edad, y que se manifiesta por tres (o más) de los hechos siguientes:

1. Incumplimiento de las normas sociales respecto a los comportamientos legales, que se manifiesta por actuaciones repetidas que son motivo de detención.

2. Engaño, que se manifiesta por mentiras repetidas, utilización de alias o estafa para provecho o placer personal.

3. Impulsividad o fracaso para planear con antelación.

4. Irritabilidad y agresividad, que se manifiesta por peleas o agresiones físicas repetidas.

5. Desatención imprudente de la seguridad propia o de los demás.

6. Irresponsabilidad constante, que se manifiesta por la incapacidad repetida de mantener un comportamiento laboral coherente o cumplir con las obligaciones económicas.

7. Ausencia de remordimiento, que se manifiesta con indiferencia o racionalización del hecho de haber herido, maltratado o robado a alguien.

B. El individuo tiene como mínimo 18 años.

C. Existen evidencias de la presencia de un trastorno de la conducta con inicio antes de los 15 años.

D. El comportamiento antisocial no se produce exclusivamente en el curso de la esquizofrenia o de un trastorno bipolar. (Manual DSM-v, numeral 301.7, 2014, p. 659).

Este trastorno de la personalidad suele asociarse a ciertas anormalidades estructurales y funcionales en la corteza prefrontal de la persona y su amígdala cerebral. En específico, la corteza orbitofrontal del cerebro está relacionada con el control de impulsos y la planificación. Por otra parte, la amígdala cerebral hace parte esencial del sistema límbico, esto es, de las emociones. Incluso, la amígdala es fundamental para identificar y actuar frente a situaciones de riesgo y miedo. Es menester, en este punto, agregar una perspectiva conjunta con la neurociencia.

Para García-López (2019), "la relación entre togas negras (expertos en derecho) y batas blancas (expertos en psicología) tiene una fascinante historia” (p. 102). El neuroderecho; es decir, el estudio de la relación entre el derecho y las neurociencias ha despertado en los últimos años un gran interés social (Ariano, 2016). Taylor et al. (1991), analizaron los roles desempeñados por los neuropsicólogos (NP) y una nueva categoría de abogados: los neurolawyers (NLS) o neuroabogados-neurojuristas. En Estados Unidos las neurociencias cada vez asumen un rol más indiscutible: el 5 por ciento de los juicios por homicidio y más del 25 por ciento de los 
juicios de pena capital utilizan las neurociencias para decidir sobre la severidad de la pena, la insanidad mental o un castigo atenuado (Presidential Commission for the Study of Bioethical Issues, 2015).

La Comisión Presidencial para el Estudio de Asuntos Bioéticos de Estados Unidos reseñó en su informe "Gray Matters V2" un caso interesante en el que la neurociencia ha influido en las decisiones judiciales. En People v. Chiesa. n. ${ }^{\circ}$ co-47001, 2005, un hombre acusado por dos homicidios, a pesar de que estaba consciente de lo que estaba haciendo cuando les disparó a dos de sus vecinos, su conducta fue guiada principalmente por impulso, no por elección. A pesar de la planificación evidente, notificar a la policía de su plan homicida, conducir su camioneta sin problema y apuntar con su arma a dos individuos diferentes: el jurado lo condenó por el delito menor de asesinato en segundo grado en lugar de asesinato premeditado de primer grado. ¿La razón? La neurociencia. En dicho caso se utilizó las tecnologías de tomografía asistida por computadora (CAT), la tomografía por emisión de positrones (PET) y la tomografía computarizada por emisión de un solo fotón (SРECT), que revelaron daños en su corteza prefrontal, lóbulos temporales y cerebelo, daño que según los expertos afectaría su control de los impulsos y su temperamento, el jurado aceptó el argumento.

De acuerdo con Gerben Meynen, es posible identificar tres áreas básicas de investigación en neuroderecho: revisión, evaluación e intervención. El dominio de revisión cubre la investigación sobre la necesidad que surge de los hallazgos neurocientíficos, de revisar o incluso eliminar partes de la Ley y las prácticas jurídicas. Sobre la evaluación, Meynen (2014) se refiere a las evaluaciones del estado mental y cerebral de individuos, probablemente acusados y presos, pero potencialmente también miembros del jurado. En el futuro, los cerebros de las personas pueden analizarse utilizando técnicas neurocientíficas para responder preguntas, por ejemplo, sobre mentir (acusados), locura (acusados), riesgo de reincidencia (acusados y prisioneros), o sesgo (posibles miembros del jurado) (Greely, 2013, citado en Meynen, 2014). El tercer dominio se refiere a todo tipo de intervenciones basadas en el cerebro y relacionadas con la Ley. Se destacan tres tipos de intervención: tratamiento, mejora y manipulación (Meynen, 2014). Estamos avanzando hacia un futuro en el que lo artificial y lo orgánico interactuarán más directamente que nunca, por esto debemos analizar cuáles son los riesgos que estamos dispuestos a asumir, y si hay caminos en este territorio desconocido a los que no deseamos ingresar (Müller y Rotter, 2017).

Retomando el enlace entre trastornos mentales y delincuencia sexual, José Ignacio Ruiz, en la gran compilación de García-López, relaciona que, en general, se vincula con parafilias, desórdenes de personalidad y psicopatía (Ruiz, 2014). Menciona, por ejemplo, los estudios que relacionan puntajes elevados en psicopatía con la reincidencia (Ruiz, 2014). La mayoría de las personas con el trastorno de la personalidad antisocial presentan, como se mencionó anteriormente, varias disfuncionalidades en su estructura y funcionamiento cerebral. En la investigación de Raine et al. (2000), en el grupo analizado con el trastorno, encontraron una reducción 
del $11,0 \%$ en el volumen de materia gris prefrontal y también una actividad autonómica reducida (conductancia de la piel y frecuencia cardíaca) durante un estresor social en el que los participantes dieron un discurso grabado en video sobre sus fallas. Por otra parte, al analizar el mapa de las conexiones entre las neuronas del cerebro, también es posible encontrar interrupción funcional (Jiang et al., 2017). Al seguir a Pemment (2012), no existe un solo factor asociado a este trastorno, más bien, un conjunto de ellos. Lo cierto es que, como explican Glenn, Yang y Raine:

Los estudios de imágenes cerebrales están comenzando a proporcionar evidencia de déficits neurobiológicos en jóvenes y adultos con comportamiento antisocial y rasgos similares a los psicópatas. Se han identificado numerosas regiones cerebrales involucradas en procesos sociales. Con mayor frecuencia, se han identificado anomalías cerebrales en la amígdala y la corteza prefrontal orbitofrontal y ventromedial, que desempeñan papeles clave en el condicionamiento del miedo, la socialización y la integración de la emoción en la toma de decisiones. También se han implicado regiones adicionales involucradas en el proceso de juicio moral. (Glenn et al., 2012).

Frente a las cifras de prevalencia del trastorno de la personalidad antisocial y las psicopatías en cárceles, es necesario adoptar una postura crítica. En primer lugar, las estadísticas aportadas son reprochadas por ser sobrediagnósticos de TAP en prisiones. Rotter et al., en su artículo Trastornos de personalidad en prisión: ¿Acaso no son todos antisociales?, revisaron 12 estudios que relacionan la prevalencia en prisiones y encontraron que el trastorno de personalidad antisocial en los hombres se halló desde el $11 \%$ al 78 \% de las personas en prisiones (Rotter et al., 2002), cifras que no son consistentes y demasiado dispersas. Es imperativo que los médicos comprendan e incorporen un conocimiento del entorno penitenciario en su evaluación mental. Tómese por ejemplo el primer de siete criterios diagnósticos, el cual, siguiendo el DSM-v, dice: "Incumplimiento de las normas sociales respecto a los comportamientos legales, que se manifiesta por actuaciones repetidas que son motivo de detención" (APA, 2014, p. 659). Pregunto, ¿acaso no todas las personas que se encuentran privadas de su libertad han incumplido normas sociales y se encuentran detenidas? El hecho es que es necesario partir de que las personas privadas de su libertad lo están por haber quebrantado normas jurídicas y haber incurrido en conductas antisociales, por lo que las cifras que relacionan la prevalencia de este tipo de trastornos pueden estar sobredimensionando la realidad. Incluso, resulta relevante diferenciar los rasgos de personalidad de los trastornos de personalidad: los rasgos son parte de la identidad subjetiva de cada persona; sin embargo, es un trastorno cuando se vuelven incompatibles con las relaciones interpersonales, laborales, académicas, la autopercepción entre otras, que generan sufrimiento a quien la padece (APA, 2014).

Los comportamientos contra la vida, integridad personal, libertad y formación sexuales contra niños, niñas y adolescentes, no necesariamente están relacionados con una enfermedad mental o con un trastorno de personalidad o psicopatía. Estudios de criminología sexual, 
enfocados en aspectos de aprendizaje criminal, psicológicos, sociales o biológicos indican, entre otros, los siguientes aspectos:

- El uso de comportamientos violentos frecuentes y el aprendizaje de los beneficios que se obtienen de ello.

- Complejos que limitan su identidad sexual, lo que puede propiciar el interés en la exploración de diversos estímulos sexuales para superar la situación.

- Exigencias socioculturales que imponen estereotipos asociados a un impulso sexual fuerte, olvidando los aspectos sentimentales, el respeto y los compromisos sociales. (Gómez y Juárez, 2014, p. 151).

El Centro de Investigación en Política Criminal de la Universidad Externado de Colombia, con su reciente publicación sobre prisión perpetua y su inviabilidad (Pardo et al., 2019), relaciona también el informe de la Defensoría del Pueblo "Violencias basadas en género y discriminación” del año 2018, en el que claramente se establece que dentro de las causas de las violencias se encuentran condiciones socioculturales tales como la dependencia económica, bajos grados de escolaridad, la desprotección laboral y las prácticas culturales machistas y patriarcales, que se replican no solo en el ámbito familiar sino también en el ámbito público (Defensoría del Pueblo, 2018).

Lo cierto es que gran parte de los factores que podrían conducir a la violencia contra niños, niñas y adolescentes, están lejos de ser asuntos exclusivos de la psicopatología. La violencia intrafamiliar, las lesiones y homicidios contra menores, parecen ser cuestiones más de cultura, relaciones de control y poder, machismo y prevención (García, 2013), que tan solo de salud mental. Por su parte, los delitos sexuales no están causalmente relacionados con padecimientos mentales o psicopatías. Es decir, nada es óbice para analizar criminológicamente los comportamientos contrarios a la infancia y adolescencia. Una vez se entienda científicamente el problema, en adelante sí se podrían plantear propuestas serias que puedan conjurar las causas y brindar una respuesta real a la problemática.

Por otra parte, las conductas victimizantes relacionadas con personas que padecen algún trastorno mental, no excluyen el posible tratamiento, sea con miras de curar un padecimiento mental, o de intentar ajustar los comportamientos a lo que socialmente se espera (Borbón, 2020). Tanto mediante un abordaje psicológico, como farmacológico, es preferible a tener que renunciar a un ciudadano que conserva su dignidad humana y no debe ser discriminado o estigmatizado por el padecimiento de una enfermedad mental (Borbón, 2020). Inclusive, en los trastornos antisociales de personalidad, o en psicopatologías, en las que se cree que la persona es incurable, esto no parece ser del todo cierto. La terapia cognitivo-conductual ha mostrado algunos resultados positivos en pacientes adultos, pues está dirigida a ajustar rasgos de personalidad como la impulsividad o la agresividad, o las dificultades interpersonales y puede ayudar a reducir las conductas desafiantes (NICE, 2015). 
Hanson et al. (2002) publicaron una de las primeras grandes investigaciones de revisión sobre la efectividad del tratamiento psicológico para delincuentes sexuales, comparando con población penitenciaria que no recibe tratamiento:

Promediado en todos los estudios, la tasa de reincidencia de delitos sexuales fue menor para los grupos de tratamiento (12.3\%) que para los grupos de comparación ( $16.8 \%, 38$ estudios, promedio no ponderado). Se encontró un patrón similar para la reincidencia general, aunque las tasas generales fueron previsiblemente más altas (tratamiento $27.9 \%$, comparación $39.2 \%$, 30 estudios). Los tratamientos actuales (cognitivo-conductual, $\mathrm{k}=13$; sistémico, $\mathrm{k}=2$ ) se asociaron con reducciones tanto en la reincidencia sexual (del 17,4 al 9,9\%) como en la reincidencia general (del 51 al $32 \%$ ). (Hanson et al., 2002, p. 169).

Harrison et al., publicaron un metaanálisis que relaciona la participación de un total de 12 811 delincuentes sexuales. La conclusión fue:

Este meta-análisis ha encontrado apoyo para la proposición de que los programas de tratamiento cognitivo conductual para delincuentes sexuales son un método efectivo para reducir la reincidencia. En general, los resultados de los análisis demuestran que los programas cognitivo-conductuales son efectivos para reducir los comportamientos de reincidencia sexual y violenta combinada después del tratamiento. Curiosamente, estos resultados están respaldados por varios meta-análisis anteriores que encontraron que los delincuentes sexuales tratados exhiben niveles más bajos de reincidencia sexual. (Harrison et al., 2020, p. 17).

Incluso, en el mismo artículo, se concluye que los delincuentes sexuales son más propensos a reincidir cometiendo delitos no sexuales en lugar de delitos sexuales. Es importante mencionar que el tratamiento puede reducir las tasas de reincidencia, pero no proporciona una cura en estricto sentido (Brooks-Gordon et al., 2006).

En el año 2003, Burns et al., analizaron el caso de un hombre de 40 años, aparentemente normal, que desde el año 2000 adquirió una desenfrenada adicción e interés hacia los servicios sexuales, la pornografía, y la pornografía infantil. Incluso, le hizo insinuaciones sexuales inadecuadas a su hijastra. Anterior a ese año, no se registraba problema similar, ni conflictos maritales por esa razón. Fue condenado y enviado a rehabilitación, donde fue expulsado por solicitar servicios sexuales del personal de salud. El historial médico del paciente relacionaba un trauma en su cabeza, ocurrido 16 años antes, que en ese entonces le produjo migrañas e hipertensión por dos años. Mediante la utilización de tecnología de resonancia magnética se descubrió un tumor de significativo tamaño cubierto por una porción quística en su cerebro. Este presionaba la corteza orbitofrontal derecha y distorsionaba su corteza prefrontal dorsolateral (Burns et al., 2003).

Al mencionado paciente le realizaron una resección tumoral exitosamente. En los días posteriores a la operación, recobró la normalidad y participó satisfactoriamente en un programa de Sexaholics Anonymous. Siete meses después, ya no se consideraba una amenaza para su hijastra y pudo volver a su hogar. Sin embargo, en octubre de 2001, desarrolló una migraña persistente 
y comenzó a colectar pornografía de manera secreta. Nuevamente se realizó un análisis con resonancia magnética y se confirmó que el tumor había vuelto a crecer. En febrero de 2002 se realizó nuevamente la resección tumoral, con resultados positivos (Burns et al., 2003). Este caso, en nuestro concepto, demuestra la importancia que en la toma de decisiones judiciales debe tener la neurociencia. A todas luces, si planteamos que una persona con un problema neurológico similar hubiese sido condenada a prisión perpetua, sería esa una sentencia injusta que no tuvo consideración por el cerebro y el estado mental del procesado; su culpabilidad.

Lo cierto es que no existe la "intratabilidad" de una persona que padece una enfermedad mental, como tampoco existe la delincuencia por "naturaleza”. El cerebro es plástico, cambiante, moldeable, se ajusta a sus circunstancias. Si bien es en la infancia en la que más periodos críticos de neuroplasticidad existen (Purves, Augustine y Fitzpatrick, 2016), ello no es óbice para que a lo largo de toda la vida humana el cerebro conserve su capacidad neurobiológica de cambiar.

Los avances en las neurociencias incluso nos indican que el enfoque político criminal debería estar principalmente dirigido a la prevención. Interviniendo tempranamente a la posible víctima, como también a la población en riesgo de desarrollar algún trastorno mental (Borbón, 2020). Las situaciones victimizantes en la infancia, como traumas producto del abandono parental o la agresión física, someten al cerebro del infante a niveles de estrés que eventualmente podrían desarrollarle distintos tipos de padecimientos mentales. El psiquiatra estadounidense George L. Engel, en su modelo biopsicosocial, relaciona la importancia de los factores biológicos, como la genética; los psicológicos, como emociones y pensamientos, y sociales, como la educación, pobreza y oportunidades (Borrell, 2002). En otras palabras, no existe el "delincuente nato", así como tampoco existen las personas intratables. Toda persona diagnosticada con un trastorno mental puede ser sujeto de un tratamiento psicológico o psicofarmacológico, e independientemente de la posibilidad de "curar", la dignidad humana que conserva debe ser vinculada a procurar reducir clínicamente el sufrimiento que su condición le puede estar causando.

Así pues, es necesario implementar políticas integrales de prevención de situaciones victimizantes desde la infancia. De la misma manera, la neurociencia nos puede indicar la importancia de propender por el bienestar psicológico de los menores, especialmente porque en la infancia están presentes los periodos críticos de neuroplasticidad; esto es, que el cerebro del menor es especialmente moldeable o cambiante. De esa forma, con políticas públicas dirigidas a la intervención en la familia, en la víctima y posible victimario y en el tratamiento penitenciario psicológico, es posible buscar soluciones más idóneas a la prisión perpetua, que en nada aporta una solución.

Independiente de la posibilidad de revisión de la Sentencia, el proyecto de Acto Legislativo de prisión perpetua nació con base a consideraciones peligrosistas y simbólicas. La revisión de una sentencia de prisión perpetua no garantiza el respeto por el valor intrínseco del ser humano y se le somete a consideraciones arbitrarias sobre su "resocialización". No es racional pensar que, sin mayor esfuerzo e inversión estatal, el privado de su libertad podrá alcanzar el "grado de 
resocialización" suficiente para que su sentencia sea revisada sin la implementación de serios programas de atención psicológica y física. Sin embargo, como se ha condenado a una persona de por vida, es probable que poca atención reciba esta persona por parte del Estado, pues, al fin y al cabo, se le ha condenado a permanecer privado de su libertad hasta su muerte. En ese sentido, la propuesta se construye bajo supuestos que niegan la dignidad humana al utilizar al ser humano como un medio, y no como un fin en sí mismo, que por el simple hecho de existir mantiene un valor que le es propio de manera intrínseca. Así como, no obedece a reglas de la lógica inferir que, enviando a un ser humano a una penitenciaría hacinada, se convertirá en una nueva y mejor persona. Si verdaderamente se quiere cumplir la función resocializadora de la pena, es momento de plantear políticas públicas serias de salud mental, especialmente focalizadas a grupos de riesgo y población privada de su libertad.

\section{Conclusiones}

Se expuso con suficiencia, en la primera sección, que la prisión perpetua no se muestra como un mecanismo idóneo para prevenir o disuadir la comisión de hechos victimizantes contra niños, niñas y adolescentes. Por otra parte, se relacionaron las razones por las que la reclusión de por vida no significa brindar mayor justicia, así como se mencionó la ya existencia de la privación de libertad perpetua en casos de concurso de delitos. Como tercer aspecto, se aportaron argumentos para sostener que no es justicia la reclusión en condiciones degradantes e inhumanas; las cárceles y penitenciarías de Colombia son los espacios menos idóneos para pretender proteger al condenado, afirmación aún más vigente en medio de la coyuntura por coronavirus.

Finalmente, en la segunda sección, se relacionaron los argumentos para desvirtuar la relación entre enfermedades mentales y violencia contra niños, niñas y adolescentes. Vincular causalmente los padecimientos de salud mental con la comisión de actos delictivos, se presenta estigmatizante y discriminador, a todas luces, una visión del comportamiento humano alejada de la ciencia. De la misma manera, se expusieron los argumentos suficientes para sostener que el tratamiento psicológico es una alternativa real, viable y eficiente para reducir la reincidencia en delitos sexuales y materializar la función de resocialización.

Lo cierto es que, si se busca proteger a los menores de situaciones victimizantes, lo mejor que se puede hacer es basarse en conocimientos científicos sobre el origen de comportamientos humanos antijurídicos, para verdaderamente brindar soluciones efectivas a problemáticas como estas. La prisión perpetua no es solución idónea, sino que representa una herramienta simbólica y política para responder a los fenómenos de opinión pública.

La pena es la imposición consciente de dolor que, por más límites constitucionales y legales, conserva una intrínseca brutalidad y lesividad. Si las normas rectoras del Código Penal colombiano exigen el cumplimiento de funciones de prevención general, prevención especial, retribución justa, protección al condenado y reinserción social, no es admisible la creación de castigos perpetuos que no las cumplen. De la misma manera, no es aceptable crear sanciones sin un sustento científico y empírico comprobable. 


\section{Referencias}

American Psychiatric Association. APA (2014). DSM-V, Manual Diagnóstico y Estadístico de los Trastornos Mentales. Bogotá, Colombia: Editorial Médica Panamericana.

Ariano, C. (2016). Reflexiones sobre el neuroderecho. Vox Juris, 32 (2), 101-106. Recuperado de https:// www.aulavirtualusmp.pe/ojs/index.php/VJ/article/view/777/

Bacigalupo, E. (2010). Filosofía e ideología de las teorías de la pena. Derecho y humanidades, 1 (16), 17 30. Recuperado de https://revistas.uchile.cl/index.php/RDH/article/view/16000/

Beccaria, C. (2015). De los delitos y de las penas. Madrid, España: Universidad Carlos III de Madrid.

Borbón Rodríguez (2020, mayo)._Prisión perpetua, funciones de la pena y neuroderecho. Recuperado de https://www.researchgate.net/publication/341495253_Prision_perpetua_funciones_de_la_pena_ y_neuroderecho

Borbón Rodríguez, D A. (2020). Neuroderecho y la falacia de la prisión perpetua desde la psicopatología. Revista Derecho Debates y Personas. Recuperado de https://www.revistaderecho.com.co/3/ index.php/es/blog-listing-2/item/110-neuroderecho-y-la-falacia-de-la-prision-perpetua-desde-lapsicopatologia

Borrell, F. (2002). El modelo biopsicosocial en evolución. Medicina Clínica, 119 (5), 175-179. Recuperado de https://altascapacidades.es/portalEducacion/html/otrosmedios/13034093_S300_es.pdf

Brooks-Gordon, B., \& Bilby, C. (2006). Psychological interventions for treatment of adult sex offenders. BMJ, 333 (7557), 5-6. [Traducción propia]. https://doi.org/10.1136/bmj.333.7557.5

Burns, J. M., \& Swerdlow, R. H. (2003). Right Orbitofrontal Tumor with Pedophilia Symptom and Constructional Apraxia Sign. Arch Neurol, 60 (3), 437-440. [Traducción propia]. https://doi.org/10.1001/ archneur.60.3.437

Carnevali Rodríguez, R. (2008). Derecho penal como ultima ratio. Hacia una política criminal racional. Ius et Praxis, 14 (1), 13-48. Recuperado de http://www.revistaiepraxis.cl/index.php/iepraxis/article/ view/319/

Christie, N. (1988). Los límites del dolor. México: Fondo de Cultura Económica.

Cita, R., \& González, I. (2017). La proporcionalidad de las penas en la legislación penal colombiana. Bogotá, Colombia: Grupo Editorial Ibáñez.

Colombia. Ley 599 de 2000. Por la cual se expide el Código Penal.

Congreso de la República de Colombia (2020). Acto Legislativo 01 de 2020.

Cordini, N. S. (2014). La finalidad de la pena es, según Kant, ¿puramente retributiva? Revista de Derecho, 43, 671-701. http://dx.doi.org/10.4067/S0718-68512014000200019

Corte IDH (2017). Cuadernillo de jurisprudencia de la Corte Interamericana de Derechos Humanos n. ${ }^{\circ}$ 9. Personas privadas de la libertad. Recuperado de https:/www.corteidh.or.cr/sitios/libros/todos/docs/ cuadernillo9.pdf

CIDH (31 de marzo de 2020) La CIDH urge a los Estados a garantizar la salud y la integridad de las personas privadas de libertad y sus familias frente a la pandemia del covid-19. [Comunicado de Prensa]. Recuperado de https://www.oas.org/es/cidh/prensa/comunicados/2020/066.asp 
Corte Suprema de Justicia de Colombia. Radicado n. ${ }^{\circ} 31895$ (2011). Magistrado ponente: José Leonidas Bustos.

Defensoría del Pueblo de Colombia (2018). Informe Defensorial: Violencias basadas en género y discriminación, Bogotá, Colombia: Agencia de los Estados Unidos para el Desarrollo Internacional (USAID/ Colombia). Recuperado de https://www.defensoria.gov.co/public/pdf/Informe\%20DefensorialViolencias-Basadas-Genero-Discriminacion.pdf

Ferrajoli, L. (1995). Derecho y razón, teoría del garantismo penal. Madrid, España: Editorial Trotta, S. A.

Fiscalía General de la Nación (2019). Censo delictivo FGN. Noviembre 2019.

Gaceta del Congreso (26 de julio de 2019): Proyecto de acto legislativo número 047, por medio del cual se modifica el artículo 34 de la Constitución Política, estableciendo la pena de prisión perpetua revisable, y se dictan otras disposiciones. Gaceta del Congreso: Senado y Cámara: Imprenta Nacional de Colombia. Recuperado de http://svrpubindc.imprenta.gov.co/senado/index2. xhtml?ent=Camara\&fec $=26-07-2019 \&$ num $=669$

García Montoya, L. (2013). Criminología y violencia familiar: Una aproximación a la violencia en el hogar a partir del estudio de las características del maltratador. (Tesis de doctorado). Centro de Investigación en Criminología Universidad de Castilla de la Mancha. Recuperado de http://hdl.handle. net/10578/3799

García-López, E., Mercurio, E., Nijdam-Jones, A., Morales, L. A., \& Rosenfeld, B. (2019). Neurolaw in Latin America: Current Status and Challenges. International Journal of Forensic Mental Health, 18 (3). [Traducción propia]. https://doi.org/10.1080/14999013.2018.1552634

Glenn, A., Yang, Y., \& Raine, A. (2012). Neuroimaging in Psychopathy and Antisocial Personality Disorder: Functional Significance and a Neurodevelopmental Hypothesis. In Neuroimaging in Forensic Psychiatry, (pp. 81-98). J.R. Simpson (Ed.). [Traducción propia]. https://doi.org/10.1002/9781119968900. ch5

Gómez Tagle López, E., \& Juárez Ríos, E. (2014). Criminología sexual. Revista IUs, 8 (34), 141-165. https://doi.org/10.35487/rius.v8i34.2014.127

Gómez, C. (2016). Introducción al derecho penal constitucional. Bogotá, Colombia: Ediciones Nueva Jurídica.

Gutiérrez Quevedo, M. (2015). Políticas públicas y prevención en Colombia. En Sierra Ramírez, C., Rosas, N., Camacho, D., Triana Sánchez, J. L. et al. Política criminal y "prevención". (pp. 21-43) Bogotá, Colombia: Universidad externado de Colombia. https://doi.org/10.4000/books.uec.1131

Gutiérrez Quevedo, M. (2017). Prólogo. Política criminal y abolicionismo, hacia una cultura restaurativa. En Gutiérrez Quevedo, M., \& Olarte Delgado, Á. M. (Eds.), Política criminal y abolicionismo, hacia una cultura restaurativa: Cátedra de Investigación Científica del Centro de Investigación en Política Criminal $N^{\circ}$ 9. (pp. 17-21).Universidad Externado de Colombia. https://doi.org/10.4000/books. uec.2281

Hanson, R. K., Gordon, A., Harris, A. \& Marques, J. K. (2002). First report of the collaborative outcome data project on the effectiveness of psychological treatment for sex offenders. Sexual abuse: A journal of research and treatment, 14(2), 169-197. https://doi.org/10.1177/107906320201400207 
Hare, R. (1991): Psychopathy Check List. [Traducción propia]. Toronto, Canadá: Multi-Health Systems.

Hare, R. D. (1 de febrero de 1996). Psychopathy and Antisocial Personality Disorder: A Case of Diagnostic Confusion. Psychiatrictimes.com (13), 2. [Traducción propia]. Recuperado de https://www.psychiatrictimes.com/view/psychopathy-and-antisocial-personality-disorder-case-diagnostic-confusion

Harrison, J.L., O’Toole, s. K., Ammen, S., Ahlmeyer, S., Harrell, S. N., \& Hernande J.L. (2020). Sexual Offender Treatment Effectiveness Within Cognitive-Behavioral Programs: A Meta-Analytic Investigation of General, Sexual, and Violent Recidivism. Psychiatry, Psychology and Law, 27 (1), 1-25. https://doi.org/10.1080/13218719.2018.1485526

Henderson, C., Evans-Lacko, S., \& Thornicroft, G. (2013). Mental illness stigma, help seeking, and public health programs. American Journal of Public Health, 103(5), 777-780. https://doi.org/10.2105/ АJPH.2012.301056

INPEC. Instituto Nacional Penitenciario y Carcelario (2020, febrero). Boletín estadístico. Recuperado de https://www.inpec.gov.co/estadisticas/-/document_library/TwBujQCwH6Kv/view/49294INPEC

INPEC. Instituto Nacional Penitenciario y Carcelario (2020, marzo). Informe estadístico. Recuperado de https://www.inpec.gov.co/estadisticas/-/document_library/TwвuJQCwH6Kv/view/49294

INPEC. Instituto Nacional Penitenciario y Carcelario. (2020, noviembre). Situación actual covid-19. Establecimientos carcelarios del orden nacional. Recuperado de https://www.inpec.gov.co/web/guest/ covid-19-establecimientos-inpec

INPEC. Instituto Nacional Penitenciario y Carcelario (16 de septiembre de 2020). Derecho de Petición con radicado No. 2020ER0105109. Peticionario: Diego Alejandro Borbón Rodríguez.

Instituto Nacional de Medicina Legal y Ciencias Forenses. (2018). Informe FORENSIS 2018 Datos para la vida. Recuperado de https://acotaph.org/assets/forensis-2018.pdf

Jiang, W., Shi, F., Liao, J., Liu, H., Wang, T., Shen, C., Hu D., Wang W., \& Shen D. (2017). Disrupted functional connectome in antisocial personality disorder. Brain Imaging and Behavior, 11 (4), 10711084. [Traducción propia]. https://doi.org/10.1007/s11682-016-9572-z

Kant, E. (1996). Metafísica de las costumbres. Barcelona, España: Altaya.

Kelsen, H. (2019). Teoría pura del derecho, 16a edición. México: Editorial Porrúa.

Kessler, R. C., Berglund, P. A., Bruce, M. L., Koch, J. R., Laska, E. M., Leaf, P. J., Manderscheid, R. W., Rosenheck, R. A., Walters, E. E., \& Wang, P. S. (2001). The prevalence and correlates of untreated serious mental illness. Health Services Research, 36(6 Pt 1), 987-1007. Recuperado de https://www.ncbi.nlm.nih.gov/pmc/articles/PMc1089274/

MacArthur Research Network on Mental Health and the Law (1998). MacArthur Community Violence Risk Study. [Traducción propia]. Recuperado de https://macarthur.virginia.edu/violence.html

Mackenzie, D. L., \& Farrington, D. P. (2015). Preventing future offending of delinquents and offenders: What have we learned from experiments and meta-analyses. Journal of Experimental Criminology, 11 (4), 565-595. [Traducción propia]. https://doi.org/10.1007/s11292-015-9244-9

Martins Valença, A., Nascimento, I., \& Egidio Nardi, A. (2013). Relationship between sexual offences and mental and developmental disorders: A review. Archives of Clinical Psychiatry, 40 (3), 97-104. [Traducción propia]. https://doi.org/10.1590/S0101-60832013000300004 
McLawsen, J. E., Scalora, M. J., \& Darrow, C. (2012). Civilly committed sex offenders: A Description and Interstate Comparison of Populations. Psychology, Public Policy, and Law, 18 (3), 453-476. [Traducción propia]. https://doi.org/10.1037/a0026116

Meynen, G. (2014). Neurolaw: Neuroscience, Ethics, and Law. Review Essay. Ethic Theory Moral Practice, 17 (4), 819-829. [Traducción propia]. https://doi.org/10.1007/s10677-014-9501-4

Ministerio de Salud y Protección Social. (2019). Sala situacional de la población adulta mayor. Recuperado de https://www.minsalud.gov.co/sites/rid/Lists/BibliotecaDigital/RIDE/DE/Ps/sala-situacionalpoblacion-adulta-mayor.pdf

Montealegre, E., \& Perdomo, J. (2006). Funcionalismo y normativismo penal. Una introducción a la obra de Günther Jakobs. Bogotá, Colombia: Universidad Externado de Colombia.

Müller, O, \& Rotter, S. (2017). Neurotechnology: Current Developments and Ethical Issues. Frontiers in Systems Neuroscience, 11 (93). 1-5. [Traducción propia]. https://doi.org/10.3389/fnsys.2017.00093

NICE. The National Institute for Health and Care Excellence. (2015). Personality disorders: Borderline and antisocial. Recuperado de https://www.nice.org.uk/guidance/qs88/resources/personality-disordersborderline-and-antisocial-pdf-2098915292869

Pardo, A., Moncayo, A., \& Olarte, A. (2019). Consideraciones sobre la inviabilidad de la prisión perpetua. Bogotá, Colombia: Centro de Investigación en Política Criminal. Universidad Externado de Colombia.

Pemment, J. (2012). The neurobiology of antisocial personality disorder: The quest for rehabilitation and treatment. Aggression and Violent Behavior, 18 (1), 79-82. [Traducción propia]. http://dx.doi. org/10.1016/j.avb.2012.10.004

Pérez, E. (2015). Psicología, derecho penal y criminología. Bogotá, Colombia: Editorial Temis.

Presidential Commission for the Study of Bioethical Issues. (2015). Gray Matters. Topics at the Intersection of Neuroscience, Ethics, and Society, Vol.2 con cifras de Farahany (2014) [Traducción propia]. Carolina del Norte, usa: Duke University.

Purves, D., Augustine, G., \& Fitzpatrck, D. (2016). Neurociencia. 5ª edición. Bogotá, Colombia: Editorial Médica Panamericana.

Raine, A., Lencz, T., Bihrle, S., LaCasse, L., \& Colletti, P. (2000). Reduced Prefrontal Gray Matter Volume and Reduced Autonomic Activity in Antisocial Personality Disorder. Archives Of General Psychiatry, 57(2), 119-127. [Traducción propia]. https://doi.org/10.1001/archpsyc.57.2.119

Real Academia Española (RAE). (s.f.). Definición de Fin. Recuperado de https://dle.rae.es/fin?m=form.

Romero Sánchez, A., \& López Cantero, E, J. (2017). Populismo punitivo, actitudes punitivas y la crisis del sistema penal en Colombia: Reflexiones y perspectivas. Revista Pluriverso, 8, 1-13. Recuperado de https://publicaciones.unaula.edu.co/index.php/Pluriverso/article/view/371

Ross, A. (1994). Sobre el derecho y la justicia. Buenos Aires, Argentina: Editorial Eudeba.

Rotter, M., Way, B., Steinbacher, M., Sawyer, D., \& Smith, H. (2002). Personality disorders in prison: Aren't they all antisocial? Psychiatric Quarterly, 73 (4), 337-349. [Traducción propia]. https://doi. org/10.1023/A:1020468117930 
Ruiz, J. (2014). Evaluación psicológica forense en acusados de delitos sexuales. En García López (Ed.), Psicopatología Forense. Comportamiento humano y tribunales de justicia. (pp. 266-288). Bogotá, Colombia: Manual Moderno.

Sánchez, C. (2010). Pena, castigo y prevención en la sociedad actual. De la pena privativa de libertad a las alternativas de la pena. Revista Digital de la Maestría en Ciencias Penales, (2), 101-148. Recuperado de https://revistas.ucr.ac.cr/index.php/RDMCP/article/view/12562

Sandel, M. (2011). Justicia. ¿Hacemos lo que debemos? Barcelona, España: Penguin Random House Grupo Editorial.

Sentencia T-153 de 1998. Corte Constitucional de Colombia. Magistrado Ponente: Eduardo Cifuentes Muñoz.

Sentencia T-388 de 2013. Corte Constitucional de Colombia. Magistrado Ponente: María Victoria Calle. Sentencia C-143 de 2015. Corte Constitucional de Colombia. Magistrado Ponente: Luis Ernesto Vargas. Sentencia T-762 de 2015. Corte Constitucional de Colombia. Magistrado Ponente: Gloria Stella Ortiz. Sentencia T-151 de 2016. Corte Constitucional de Colombia. Magistrado Ponente: Alberto Rojas Ríos. Sentencia T-208 de 2018. Corte Constitucional de Colombia. Magistrado Ponente: Diana Fajardo Rivera. Stuart, H. (2003). Violence and mental illness: an overview. World Psychiatry, 2 (2), 121-124. Recuperado de https://pubmed.ncbi.nlm.nih.gov/16946914/

Taylor, J. S., Harp, J. A., \& Elliott, T. (1991). Neuropsychologists and neurolawyers. Neuropsychology, 5(4), 293-305. [Traducción propia]. https://doi.org/10.1037/0894-4105.5.4.293 